\title{
A Numerical Simulation of Silver-Water Nanofluid Flow with Impacts of Newtonian Heating and Homogeneous-Heterogeneous Reactions Past a Nonlinear Stretched Cylinder
}

\author{
Muhammad Suleman 1,2 (D), Muhammad Ramzan ${ }^{3,4, *}$, Shafiq Ahmad ${ }^{5}$, Dianchen Lu ${ }^{1}(\mathbb{D}$, \\ Taseer Muhammad ${ }^{6}$ and Jae Dong Chung ${ }^{4}(\mathbb{D}$ \\ 1 Department of Mathematics, Faculty of Science, Jiangsu University, Zhenjiang 212013, China; \\ suleman@ujs.edu.cn (M.S.); dclu@ujs.edu.cn (D.L.) \\ 2 Department of Mathematics, Comsats University, Islamabad 44000, Pakistan \\ 3 Department of Computer Science, Bahria University, Islamabad Campus, Islamabad 44000, Pakistan \\ 4 Department of Mechanical Engineering, Sejong University, Seoul 143-747, Korea; jdchung@sejong.ac.kr \\ 5 Department of Mathematics, Quaid-i-Azam University, Islamabad 44000, Pakistan; \\ ashafiq@math.qau.edu.pk \\ 6 Department of Mathematics, Government College Women University, Sialkot 51310, Pakistan; \\ taseer_qau@yahoo.com \\ * Correspondence: mramzan@bahria.edu.pk; Tel.: +92-300-5122700
}

Received: 29 December 2018; Accepted: 29 January 2019; Published: 24 February 2019

\begin{abstract}
The aim of the present study is to address the impacts of Newtonian heating and homogeneous-heterogeneous (h-h) reactions on the flow of $\mathrm{Ag}-\mathrm{H} 2 \mathrm{O}$ nanofluid over a cylinder which is stretched in a nonlinear way. The additional effects of magnetohydrodynamics (MHD) and nonlinear thermal radiation are also added features of the problem under consideration. The Shooting technique is betrothed to obtain the numerical solution of the problem which is comprised of highly nonlinear system ordinary differential equations. The sketches of different parameters versus the involved distributions are given with requisite deliberations. The obtained numerical results are matched with an earlier published work and an excellent agreement exists between both. From our obtained results, it is gathered that the temperature profile is enriched with augmented values radiation and curvature parameters. Additionally, the concentration field is a declining function of the strength of $h-h$ reactions.
\end{abstract}

Keywords: Newtonian heating; nonlinear thermal radiation; nonlinear stretching cylinder; homogeneous/heterogeneous reactions; nanofluid

\section{Introduction}

In copious engineering processes, the role of poor thermal conductivity of certain base fluids is considered to be a big hurdle to shape a refined product. To overcome such snag, numerous practices such as clogging, abrasion, and pressure loss were engaged but the results were not very promising. The novel idea of nanofluid, which is an engineered amalgamation of metallic particles with size $(<100 \mathrm{~nm})$ and some traditional fluids like ethylene glycol, presented by Choi [1], has revolutionized the modern world. Many heat transfer applications [2] such as domestic refrigerators, microelectronics, hybrid-power engines, and fuel cells possess numerous characteristics that make them valuable because of nanofluids. In all aforementioned applications, enriched thermal conductivity is observed whenever some metallic particles are added to the ordinary base fluid [3]. The idea of a nanofluid with multiple 
dimensions has been conversed by many researchers and scientists in the last two decades. Amongst these, $\mathrm{Li}$ et al. [4] used a mixture of $\mathrm{H}_{2} \mathrm{O}-\mathrm{CuO}$ for the enhancement of the solidification rate. The finite element method is engaged to obtain the numerical solution of the problem. Sheikholeslami [5] pondered over the influence of radiation and magnetohydrodynamic on the $\mathrm{Al}_{2} \mathrm{O}_{3}-\mathrm{H}_{2} \mathrm{O}$ mixture past a spongy semi-annulus. The numerical solution of the problem is witnessed by employing the Control Volume Finite Element Method (CVFEM). The flow of Casson nanofluid with inserted multi-walled carbon nanotubes past a swirling cylinder was deliberated by Ramzan et al. [6] using bvp4c MATLAB software. The said problem is pondered with impacts of entropy generation and melting heat transfer. The flow of micropolar nanofluid with binary chemical reaction, double stratification, and activation energy is also studied by Ramzan et al. [7]. The flow of viscoelastic nanofluid with analysis of entropy generation past an exponential stretched surface was discussed by Suleman et al. [8]. Farooq et al. [9] deliberated the flow of Newtonian fluid with the amalgamation of nanoparticles by utilizing the BVPh 2.0 technique and many therein [10-15].

The role of MHD is vital in abundant fluid flows and has many applications like medicine, aerospace, nuclear reactors, MHD generators, petroleum processes, and astrophysics. The explorations highlighting the effects of MHD may include a study by Azam et al. [16] who found the numerical solution for the time-dependent MHD Cross nanofluid flow under the influence of nonlinear thermal radiation and zero mass flux conditions. The flow of MHD nanofluid with thermal diffusion and heat generation past a permeable medium over an oscillating vertical plate is perceived by Sheikholeslami et al. [17]. Makinde and Animasaun [18] examined the MHD nanofluid flow past the upper surface of a paraboloid of revolution with impacts of quartic autocatalysis chemical reaction and nonlinear thermal radiation. Lu et al. [19] debated the rotating flow of 3D MHD Maxwell fluid with a non-Fourier heat flux and binary chemical reaction using a BVP-4c MATLAB built-in technique. The analytical technique is engaged to find the multiple solutions for MHD Jeffery-Hamel flow using the KKL nanofluid model by Rana et al. [20]. The effect of generalized Fourier and Fick laws combined with temperature-dependent thermal conductivity on 3D MHD second-grade nanofluid is considered by Ramzan et al. [21]. Yuan et al. [22] established the numerical solution of MHD nanofluid flow past a baffled U-shaped enclosure by engaging the KKL technique. The 3D flow of MHD nanofluid with varied nanoparticles including $\mathrm{Fe}_{3} \mathrm{O}_{4}, \mathrm{Cu}, \mathrm{Al}_{2} \mathrm{O}_{3}$ and $\mathrm{TiO}_{2}$ and water as the base fluid past an exponential stretched surface was discussed by Jusoh et al. [23]. Some recent investigations highlighting the importance of MHD may also be found in References [24-27].

The Newtonian heating or the conjugate convective flow is termed as a direct proportionate amid the local temperature and heat transfer rate. The role of Newtonian heating is pivotal in many processes such as heat exchanger's designing, convective flows where heat is taken from the solar radiators, and conjugate heat transfer around the fins etc. The four distinct categories of the heat transfer viz. (i) conjugate boundary condition, (ii) constant or prescribed surface heat flux, (iii) Newtonian heating, and (iv) constant or prescribed surface temperature, are given by Merkin [28]. The flow of Micropolar fluid with Newtonian heating and chemical reaction past a stretching/shrinking sheet was studied by Kamran and Wiwatanapataphee [29]. Mehmood et al. [30] examined the Oldroyd-B nanofluid flow with a transverse magnetic field and Newtonian heating. The flow of Tangent hyperbolic nanofluid under the influences of Newtonian heating, MHD and bi-convection was examined by Shafiq et al. [31]. The flow of nanofluid which is an amalgamation of the base fluid (Sodium alginate) and nanoparticles (Silver, Titanium oxide, Copper and Aluminum oxide) with effects of radiation and Newtonian heating past an isothermal vertical plate are scrutinized by Khan et al. [32]. Many researchers have undertaken the impact of Newtonian heating owing to its wide-ranging practical applications [33-36].

A close review of the literature specifies that copious research is done on the subject of nanofluids with linear/nonlinear stretching sheets in comparison to the flows past the curved stretched surfaces. This topic gets even more limited if we talk about the flows of nanofluids past cylinders stretched in a nonlinear way. So, our prime goal here is to ponder the nanofluid flow past a nonlinear stretched cylinder with nonlinear thermal radiation, h-h reactions, and Newtonian heating. The nanofluid used 
here is the mixture of water and silver. The numerical simulations are conducted for the proposed problem using the Runge-Kutta method by shooting technique. To corroborate the presented results, a comparison with an already published article is done and an excellent correlation between the two results is found.

\section{Mathematical Modeling}

Here, we assume a silver-water nanofluid incompressible flow with impacts of h-h reactions, nonlinear thermal radiation and Newtonian heating over a horizontal cylinder which is stretched in a nonlinear way. The magnetic field $B=B_{0} x^{(n-1) / 2}$ is applied in the radial direction. Owing to our assumption of a small Reynolds number, the induced magnetic field is overlooked (Figure 1).

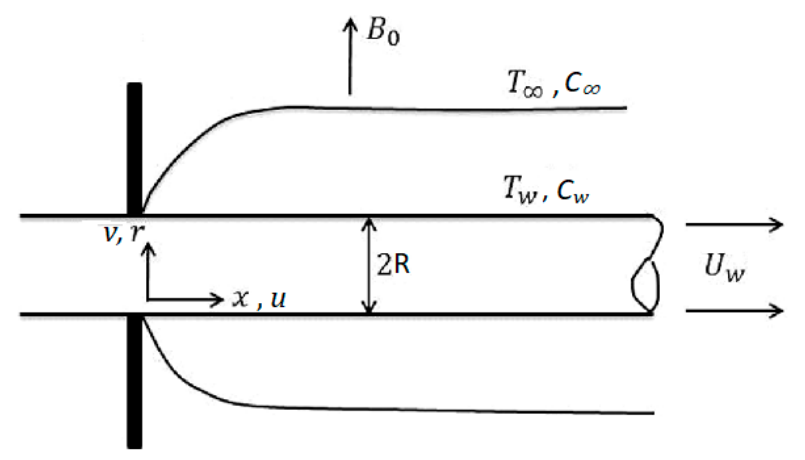

Figure 1. Diagram of the flow geometry.

The homogeneous reaction for cubic autocatalysis can be written as:

$$
\begin{gathered}
A_{1}+2 B_{1} \rightarrow 3 B_{1}, \text { rate }=k_{c} a b^{2}, \\
A_{1} \rightarrow B_{1}, \text { rate }=k_{s} a .
\end{gathered}
$$

The reaction rate dies out in the outer boundary layer. The system of boundary layer equations of the subject model is given as:

$$
\begin{gathered}
\frac{\partial(r u)}{\partial x}+\frac{\partial(r v)}{\partial r}=0 \\
u \frac{\partial u}{\partial x}+v \frac{\partial u}{\partial r}=v_{n f}\left(\frac{\partial^{2} u}{\partial r^{2}}+\frac{1}{r} \frac{\partial u}{\partial r}\right)-\frac{\sigma_{n f} B^{2}(x)}{\rho_{n f}} u \\
u \frac{\partial T}{\partial x}+v \frac{\partial T}{\partial r}=\alpha_{n f}\left(\frac{\partial^{2} T}{\partial r^{2}}+\frac{1}{r} \frac{\partial T}{\partial r}\right)-\frac{1}{\left(\rho c_{p}\right)_{n f}} \frac{\partial q_{r}}{\partial r}, \\
u \frac{\partial a}{\partial x}+v \frac{\partial a}{\partial r}=D_{A}\left(\frac{\partial^{2} a}{\partial r^{2}}+\frac{1}{r} \frac{\partial a}{\partial r}\right)-k_{1} a b^{2}, \\
u \frac{\partial b}{\partial x}+v \frac{\partial b}{\partial r}=D_{B}\left(\frac{\partial^{2} b}{\partial r^{2}}+\frac{1}{r} \frac{\partial b}{\partial r}\right)+k_{1} a b^{2},
\end{gathered}
$$

Accompanied by the conditions:

$$
\begin{aligned}
& \left.u\right|_{r=R}=U_{w}(x),\left.v\right|_{r=R}=0,\left.\frac{\partial T}{\partial r}\right|_{r=R}=h_{s} T,\left.D_{A} \frac{\partial a}{\partial r}\right|_{r=R}=k_{s} a,\left.D_{B} \frac{\partial b}{\partial r}\right|_{r=R}=-k_{s} a, \\
& \left.u\right|_{r \rightarrow \infty} \rightarrow 0,\left.T\right|_{r \rightarrow \infty} \rightarrow T_{\infty},\left.a\right|_{r \rightarrow \infty} \rightarrow a_{0},\left.b\right|_{r \rightarrow \infty} \rightarrow 0,
\end{aligned}
$$

where $U_{w}(x)=U_{0} x^{n}$, and $q_{r}=\frac{4 \sigma^{*}}{3 k^{*}} \frac{\partial T^{4}}{\partial r}=\frac{16 \sigma^{*} T^{3}}{3 k^{*}} \frac{\partial T}{\partial r}$. The numerical values of specific heat, density, and thermal conductivity of silver $(\mathrm{Ag})$ and water $\left(\mathrm{H}_{2} \mathrm{O}\right)$ are given in Table 1 . 
Table 1. Thermo-physical characteristics of the base fluid and nanoparticles $[1,37]$.

\begin{tabular}{ccc}
\hline Physical Properties & Water & Ag \\
\hline$C_{p}(\mathrm{~J} / \mathrm{kg} \mathrm{K})$ & 4179 & 235.0 \\
$\rho\left(\mathrm{kg} / \mathrm{m}^{3}\right)$ & 997.1 & $10,500.0$ \\
$K(\mathrm{~W} / \mathrm{mK})$ & 0.61300 & 429.0 \\
\hline
\end{tabular}

With the following characteristics:

$$
\begin{gathered}
\alpha_{n f}=\frac{k_{n f}}{\left(\rho C_{p}\right)_{n f}}, \\
\frac{\left(\rho C_{p}\right)_{n f}}{\left(\rho C_{p}\right)_{f}}=(1-\phi)+\phi \frac{\left(\rho C_{p}\right)_{s}}{\left(\rho C_{p}\right)_{f}}, \\
\frac{\mu_{n f}}{\mu_{f}}=\left(1.005+0.497 \phi-0.1149 \phi^{2}\right), \\
\frac{\rho_{n f}}{\rho_{f}}=1-\phi+\phi \frac{\rho_{s}}{\rho_{f}}, \\
\frac{k_{n f}}{k_{f}}=(0.9692 \phi+0.9508),
\end{gathered}
$$

The use of under-mentioned similarity transformations

$$
\begin{gathered}
\eta=\frac{r^{2}-R^{2}}{2 R} \sqrt{\frac{U_{w}}{v_{f} x}}, \psi=\sqrt{U_{w} v x} R f(\eta), \theta=\frac{T-T_{\infty}}{T_{\infty}} \\
a=a_{0} h, b=a_{0} g .
\end{gathered}
$$

Satisfy the Equation (3) and convert the Equations (4)-(7) in non-dimensional form

$$
\begin{gathered}
(1+2 \gamma \eta) f^{\prime \prime \prime}+2 \gamma f^{\prime \prime}+\left(1.005+0.497 \phi-0.1149 \phi^{2}\right)\left(1-\phi+\phi \frac{\rho_{s}}{\rho_{f}}\right)\left(\left(\frac{n+1}{2}\right) f f^{\prime \prime}-n f \prime^{2}\right) \\
-\left(1.005+0.497 \phi-0.1149 \phi^{2}\right) M f^{\prime}=0 \\
(1+2 \gamma \eta)\left(\frac{k_{n f}}{k_{f}}+\left(1+\left(N_{r}-1\right) \theta\right)^{3}\right) \theta^{\prime \prime}+\left(\frac{1}{2 K^{*}} \frac{k_{n f}}{k_{f}}+\left(1+\left(N_{r}-1\right) \theta\right)^{3}\right) \gamma \theta^{\prime}+\frac{\operatorname{Pr}}{4 K^{*}} \\
\left(1-\phi+\phi \frac{\left(\rho C_{p}\right)_{s}}{\left(\rho C_{p}\right)_{f}}\right)\left(\frac{n+1}{2} f \theta^{\prime}-n f^{\prime} \theta\right)+2(1+2 \gamma \eta)\left(N_{r}-1\right)\left(1+\left(N_{r}-1\right) \theta\right)^{2} \theta \prime^{2}=0, \\
\left.\frac{1}{S_{c}}(1+2 \gamma \eta) h^{\prime \prime}+2 \gamma h^{\prime}\right)+f h^{\prime}-\frac{2 K}{n+1} h g^{2}=0 \\
\left.\frac{1}{S_{c}}(1+2 \gamma \eta) g^{\prime \prime}+2 \gamma g^{\prime}\right)+f g^{\prime}-\frac{2 K}{n+1} h g^{2}=0
\end{gathered}
$$

Supported by the boundary conditions

$$
\begin{aligned}
& f(\eta)=0, f^{\prime}(\eta)=1, \theta^{\prime}(\eta)=-\lambda(1+\theta(\eta)), h^{\prime}(\eta)=K_{s} h(\eta), \delta g^{\prime}(\eta)=-K_{s} h(\eta) \text { as } \eta=0, \\
& \theta(\eta) \rightarrow 0, f^{\prime}(\eta) \rightarrow 0, h(\eta) \rightarrow 1, g(\eta) \rightarrow 0, \text { at } \eta \rightarrow \infty
\end{aligned}
$$

where $\lambda=h_{s}\left(v_{f} x / U_{w}\right)^{1 / 2}, M=\frac{\sigma_{f} B_{0}^{2}}{U_{0} \rho_{f}}, K^{*}=\frac{16 \sigma^{*} T_{\infty}{ }^{3}}{3 k^{*} k_{f}}, \gamma=\left(\frac{x v_{f}}{R^{2} U_{w}}\right)^{1 / 2}, N_{r}=T_{w} / T_{\infty}, K=\frac{a_{0}^{2} x k_{1}}{U_{w}}$, $K_{s}=\frac{k_{s}}{D_{A}} \sqrt{\frac{x v_{f}}{U_{w}}}$ and $S_{c}=\frac{v_{f}}{D_{A}}$.

Here, it is expected that $A_{1}$ and $B_{1}$ are equivalent. From this assumption, it is inferring that $D_{A}$ and $D_{B}$ (diffusion coefficients) are equal i.e., $\delta=1$, and on account of this supposition, we have

$$
g(\eta)+h(\eta)=1
$$


Equations (17) and (18) after the use of Equation (20) and the relevant boundary conditions take the shape

$$
\begin{gathered}
\frac{1}{S_{c}}\left((1+2 \gamma \eta) h^{\prime \prime}+2 \gamma h^{\prime}\right)+f h^{\prime}-\frac{2 k_{1}}{n+1} h(1-h)^{2}=0, \\
h^{\prime}(0)=k_{2} h(0), h(\infty) \rightarrow 1 .
\end{gathered}
$$

The physical quantities like Skin friction factor and Local Nusselt number in non-dimensional form are labelled as

$$
C_{f}=\frac{2 \tau_{w}}{\rho_{f} u_{w}^{2}}, N u_{x}=\frac{x q_{w}}{k_{f}\left(T_{f}-T_{\infty}\right)},
$$

With $\tau_{w}$ and $q_{w}$ given by

$$
\tau_{w}=\left.\mu_{n f} \frac{\partial u}{\partial r}\right|_{r=R}, q_{w}=-\left(\frac{\partial T}{\partial r}\right)_{r=R} k_{n f}+\left(q_{r}\right)_{r=R} .
$$

Equation (23), after the use of Equations (14) and (24), takes the form

$$
\begin{aligned}
& \operatorname{Re}_{x}{ }^{1 / 2} C_{f}=\left(\frac{1}{1.005+0.497 \phi-0.1149 \phi^{2}}\right) f^{\prime \prime}(0), \\
& \operatorname{Re}_{x}{ }^{-1 / 2} N u_{x}=\frac{k_{n f}}{k_{f}} \lambda\left(1+\frac{1}{\theta(0)}\right) .
\end{aligned}
$$

\section{Numerical Scheme}

The numerical solution of Equations (15), (16) and (21) supported by the boundary conditions (19) and (22) is found by the Shooting scheme. In the calculation of the numerical solution of the problem, the second and third order differential equations are transformed to first order with the help of new parameters. The selection of the initial guess estimate is pivotal in the Shooting scheme as it needs to satisfy the equation and the boundary conditions asymptotically. We have selected the tolerance as $10^{-7}$ for this specific problem. The first order system obtained in this regard is appended below:

$$
\begin{aligned}
& f(\eta)=y(1) \\
& f^{\prime}(\eta)=y(2) \\
& f^{\prime \prime}(\eta)=y(3), \\
& f^{\prime \prime \prime}(\eta)=F(3)=-\frac{1}{(1+2 \gamma \eta)}\left[\begin{array}{c}
\left(1.005+0.497 \phi-0.1149 \phi^{2}\right)\left(1-\phi+\phi \frac{\rho_{s}}{\rho_{f}}\right)\left(\left(\frac{n+1}{2}\right) f f^{\prime \prime}-n f^{2}\right) \\
+2 \gamma f^{\prime \prime}-\left(1.005+0.497 \phi-0.1149 \phi^{2}\right) M f^{\prime}
\end{array}\right], \\
& \theta(\eta)=y(4) \\
& \theta^{\prime}(\eta)=y(5)
\end{aligned}
$$

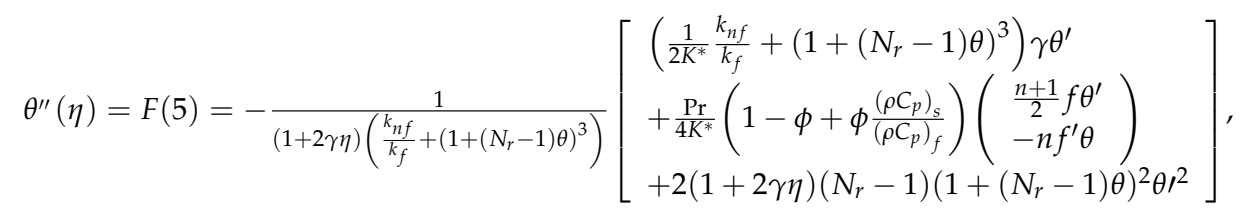

$$
\begin{aligned}
& h(\eta)=y(6) \text {, } \\
& h^{\prime}(\eta)=y(7) \\
& h^{\prime \prime}(\eta)=F(7)=-\frac{1}{(1+2 \gamma \eta)}\left[2 \gamma h^{\prime}+S_{c} f h^{\prime}-S_{c} \frac{2 k_{1}}{n+1} h(1-h)^{2}\right] \text {, }
\end{aligned}
$$


and the boundary condition becomes

$$
\begin{gathered}
y_{0}(1)=0, y_{0}(2)=1, \mathrm{y}_{0}(5)=-\lambda\left(1+y_{0}(4)\right), \mathrm{y}_{0}(7)=k_{2} y_{0}(6), \\
y_{\mathrm{inf}}(2) \rightarrow 0, y_{\mathrm{inf}}(4) \rightarrow 0, y_{\mathrm{inf}}(6) \rightarrow 0 .
\end{gathered}
$$

We have chosen $\eta_{\infty}=6$, that guarantees every numerical solution's asymptotic value accurately. Here, Table 2 depicts the comparative estimates of the present model with Qasim et al. [38] in the limiting case. Both results are found in an excellent correlation.

Table 2. Nusselt number $\left(\operatorname{Re}_{x}{ }^{-1 / 2} N u_{x}\right)$ for numerous estimates of $\gamma$ and Pr with $M=0, \phi=0.0$, $\lambda=0.0$.

\begin{tabular}{cccc}
\hline \multirow{2}{*}{$\gamma$} & $\boldsymbol{P r}$ & \multicolumn{2}{c}{$\operatorname{Re}_{x}{ }^{-1 / 2} N u_{x}$} \\
\cline { 3 - 4 } & & Qasim et al. [38] & Present Result \\
\hline \multirow{3}{*}{0.0} & 1.0 & 1.23664 & 1.236651 \\
& 6.7 & 1.00000 & 1.000000 \\
& 10 & 0.33330 & 0.333310 \\
& 0.72 & 0.26876 & 0.268770 \\
\hline \multirow{3}{*}{1.0} & 1.0 & 0.87018 & 0.870190 \\
& 6.7 & 0.74406 & 0.744070 \\
& 10 & 0.29661 & 0.296620 \\
& & 0.24217 & 0.242180 \\
\hline
\end{tabular}

\section{Results and Discussion}

In this section, we have plotted the Figures $2-11$ that exhibit the influences of various parameters like volume fraction $(0.0 \leq \phi \leq 0.3)$, magnetic parameter $(1.0 \leq M \leq 4.0)$, nonlinearity exponent $(1.0 \leq n \leq 5.0)$, curvature parameter $(0.0 \leq \gamma \leq 0.4)$, conjugate parameter $(0.4 \leq \lambda \leq 0.7)$, radiation parameter $\left(0.7 \leq K^{*} \leq 1.0\right)$, strength of homogeneous reaction $\left(0.1 \leq \kappa_{1} \leq 1.8\right)$, strength of heterogeneous reaction $\left(0.1 \leq \kappa_{2} \leq 1.8\right)$ and Schmidt number $\left(3.0 \leq S_{c} \leq 4.5\right)$ on involved distributions. Figures $2-5$ are drawn to portray the influence of $\phi, M, n$ and $\gamma$ on axial velocity. The impact of $\phi$ is discussed in Figure 2. For growing values of $\phi$, the axial velocity also augments. In Figure 3, the influence of $M$ versus the velocity field is debated. It is perceived that the velocity field deteriorates for escalated values of $M$. The Lorentz force is enforced by the strong magnetic field that hinders the fluid's velocity. Figure 4 illustrates the impression of $n$ on the axial velocity. Reduced velocity is witnessed for larger values of $n$. This is because higher values of $n$ create more collision between the particles of the fluid that hinder the fluid flow and feeble velocity if perceived. In Figures 5 and 6 , the behavior of the velocity and temperature fields for increasing values of $\gamma$ is given. It is seen that the velocity and temperature of the fluid augment for growing estimates of $\gamma$. Larger values of $\gamma$ mean a smaller radius, comparatively minimum contact region of the cylinder with the fluid and increased heat transport. That is why augmented velocity and temperature are witnessed. Figure 7 is drawn to depict the relation between the temperature of the fluid and the $\lambda$. It is detected that temperature enhances for improved values of $\lambda$. In fact, the sturdier heat transfer process is observed for larger values of $\lambda$ as more heat is moved from the cylinder to the fluid. Remembering that $\lambda=0$ means the insulated walls and $\lambda \rightarrow \infty$ constant wall temperature. Figures 8 and 9 are sketched to elaborate the influences of $K^{*}$ and $M$ on the temperature field. It is clearly perceived that temperature enhances when both $K^{*}$ and $M$ increase. The decrease in the mean absorption coefficient represents an enriched heat transfer rate and ultimately temperature is enhanced. Similarly, a stronger Lorentz force hinders the movement of the fluid, thus causing more collision between the molecules of the fluid that turns into the improved temperature. In Figures 10 and 11, the behavior of the concentration profile versus $h-h$ reactions is depicted. The concentration diminishes for growing values of $h-h$ reactions. 


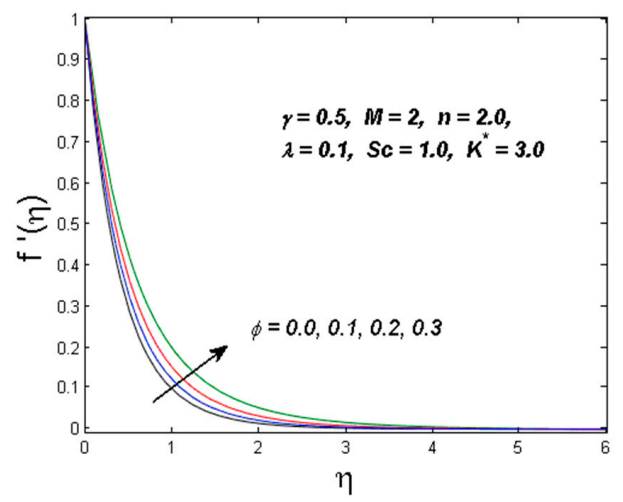

Figure 2. Diagram of axial velocity versus $\phi$.

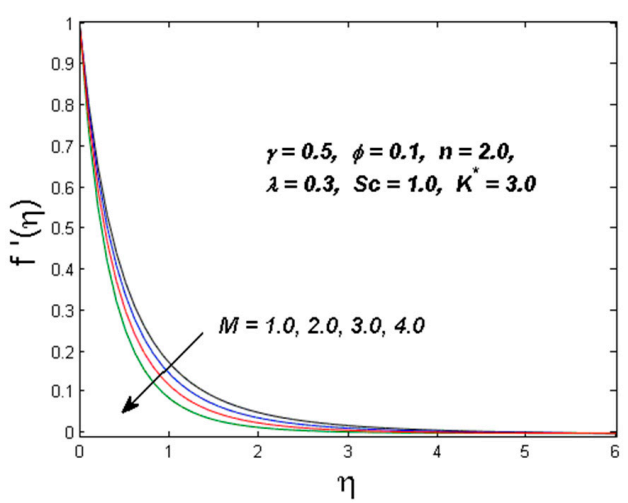

Figure 3. Diagram of axial velocity versus $M$.

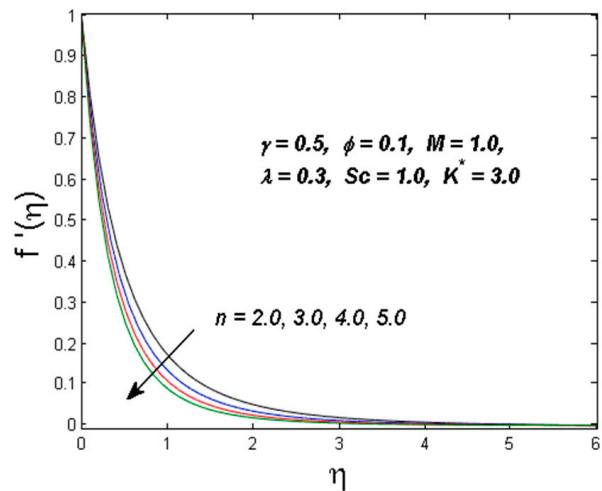

Figure 4. Diagram of axial velocity versus $n$.

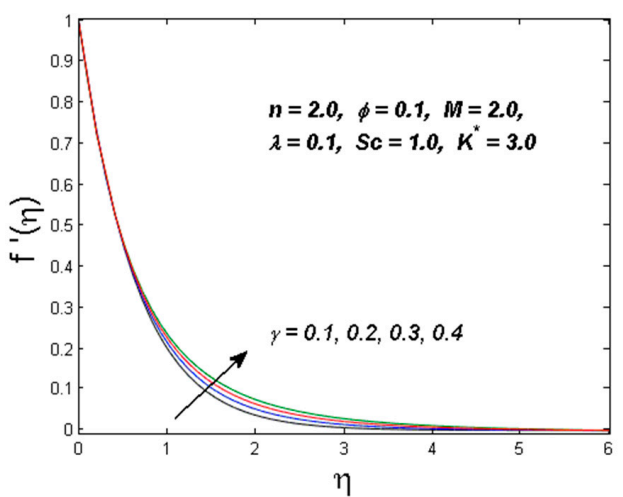

Figure 5. Diagram of axial velocity versus $\gamma$. 


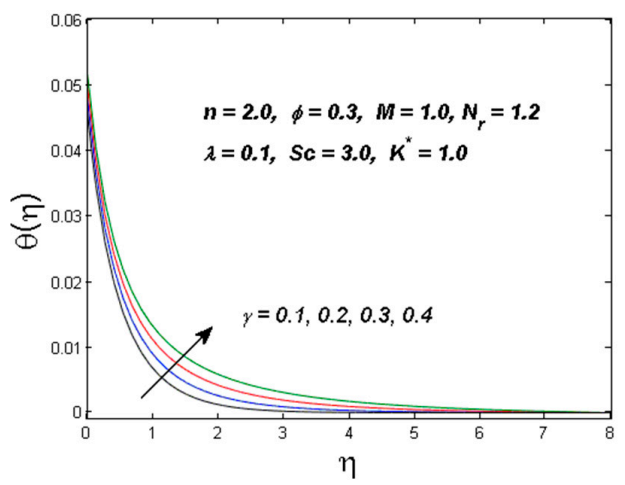

Figure 6. Diagram of the temperature field versus.

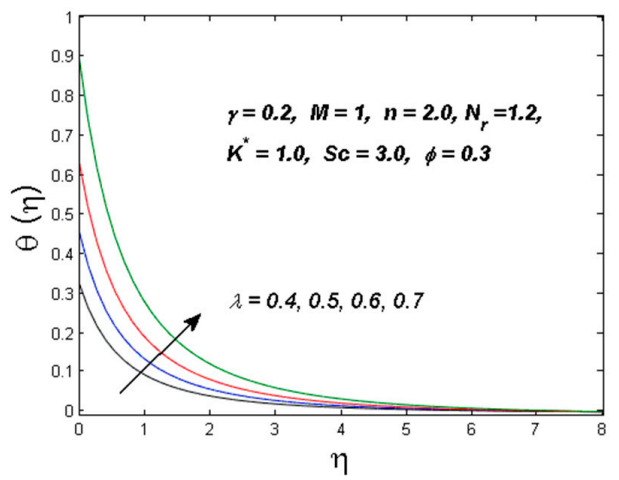

Figure 7. Diagram of the temperature field versus $\lambda$.

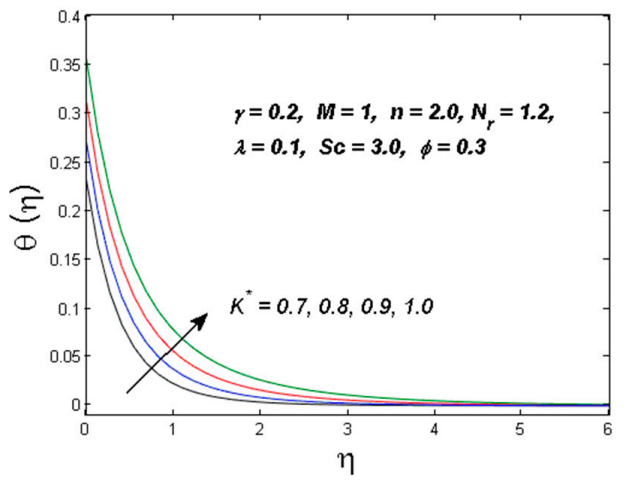

Figure 8. Diagram of the temperature field versus $K^{*}$.

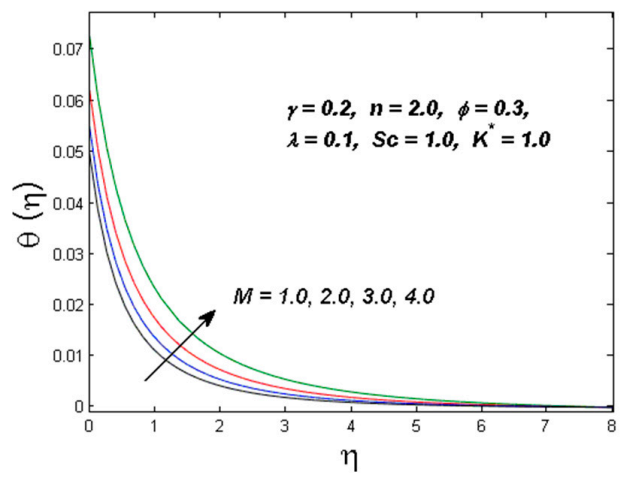

Figure 9. Diagram of the temperature field versus $M$. 


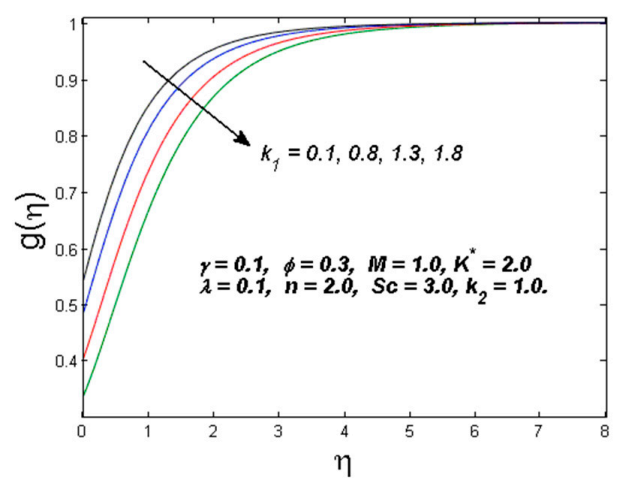

Figure 10. Diagram of concentration field versus $k_{1}$.

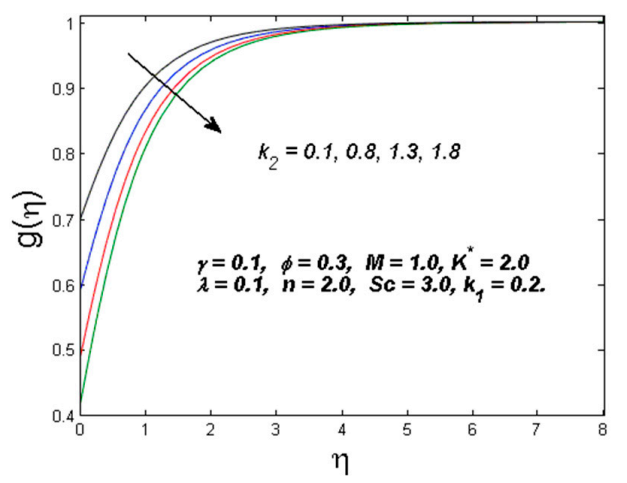

Figure 11. Diagram of concentration field versus $k_{2}$.

Table 3 depicts the numerical values of skin friction $\left(-\operatorname{Re}_{x}{ }^{1 / 2} C_{f}\right)$ and local Nusselt number $\left(\operatorname{Re}_{x}{ }^{-1 / 2} N u_{x}\right)$ for numerous estimates of thee parameters. It is perceived that skin friction and Nusselt number upsurge for growing values of solid volume friction $\phi$ and $M$ (magnetic parameter). It is also observed that for escalated values of curvature parameter $\gamma$ and nonlinearity parameter $n$, both skin friction and local Nusselt number show opposite behavior. Moreover, Skin friction and local Nusselt number exhibit a constant trend for values of the temperature ratio parameter $N_{r}$ and radiation parameter $K^{*}$.

Table 3. Numerical values of $-\operatorname{Re}_{x}{ }^{1 / 2} C_{f}$ and $\operatorname{Re}_{x}{ }^{-1 / 2} N u_{x}$ for $A g-$ water with $\operatorname{Pr}=6.2$.

\begin{tabular}{cccccccc}
\hline $\mathbf{n}$ & $\boldsymbol{\gamma}$ & $\boldsymbol{\phi}$ & $\mathbf{M}$ & $\mathbf{N}_{\mathbf{r}}$ & $\mathbf{K}^{*}$ & $-\mathbf{R e}_{x}{ }^{1 / 2} \boldsymbol{C}_{f}$ & $\mathbf{R e}_{\boldsymbol{x}}{ }^{-1 / 2} \boldsymbol{N} \boldsymbol{u}_{\boldsymbol{x}}$ \\
\hline 1.0 & 0.1 & 0.1 & 1.0 & 1.2 & 1.0 & 1.92700 & 0.32149 \\
2.0 & 0.1 & 0.1 & 1.0 & 1.2 & 1.0 & 2.45720 & 0.30963 \\
3.0 & 0.1 & 0.1 & 1.0 & 1.2 & 1.0 & 2.89050 & 0.30305 \\
2.0 & 1.0 & 0.1 & 1.0 & 1.2 & 1.0 & 2.81410 & 0.28200 \\
2.0 & 2.0 & 0.1 & 1.0 & 1.2 & 1.0 & 3.18730 & 0.26410 \\
2.0 & 3.0 & 0.1 & 1.0 & 1.2 & 1.0 & 3.54330 & 0.25369 \\
2.0 & 1.0 & 0.1 & 1.0 & 1.2 & 1.0 & 2.81410 & 0.28200 \\
2.0 & 1.0 & 0.2 & 1.0 & 1.2 & 1.0 & 3.42830 & 0.31250 \\
2.0 & 1.0 & 0.3 & 1.0 & 1.2 & 1.0 & 4.04270 & 0.34319 \\
2.0 & 1.0 & 0.1 & 1.0 & 1.2 & 1.0 & 2.81410 & 0.28200 \\
2.0 & 1.0 & 1.0 & 2.0 & 1.2 & 1.0 & 2.99960 & 0.28387 \\
2.0 & 1.0 & 1.0 & 3.0 & 1.2 & 1.0 & 3.17070 & 0.28553 \\
2.0 & 1.0 & 1.0 & 1.0 & 0.1 & 1.0 & 2.81410 & 0.28200 \\
2.0 & 1.0 & 1.0 & 1.0 & 0.7 & 1.0 & 2.81410 & 0.28200 \\
2.0 & 1.0 & 1.0 & 1.0 & 1.0 & 1.0 & 2.81410 & 0.28200 \\
2.0 & 1.0 & 1.0 & 1.0 & 1.2 & 1.0 & 2.81410 & 0.28200 \\
2.0 & 1.0 & 1.0 & 1.0 & 1.2 & 2.0 & 2.81410 & 0.28200 \\
2.0 & 1.0 & 1.0 & 1.0 & 1.2 & 3.0 & 2.81410 & 0.28200 \\
\hline
\end{tabular}




\section{Final Comments}

In the present exploration, we have pondered over the nanofluid flow (with base fluid water and nanoparticles as silver) past a nonlinear stretching cylinder with impacts of $h$-h reactions and nonlinear thermal radiation. Additional effects of Newtonian heating and magnetohydrodynamics have also been taken into account. A numerical solution of the dimensionless mathematical model is achieved via the shooting scheme. The core outcomes of the present study are as follows:

- The temperature profile is a growing function of radiation and magnetic parameters.

- For larger values of the curvature parameter, augmented velocity is observed.

- The concentration of the fluid decreases for growing values of homogeneous-heterogeneous reactions.

- For escalated values of the magnetic parameter, velocity and temperature distributions show the opposite trend.

- The skin friction and local Nusselt number show opposite behavior for curvature and nonlinearity parameters.

Author Contributions: Data curation, J.D.C.; Formal analysis, S.A.; Funding acquisition, M.S.; Investigation, D.L.; Methodology, T.M.; Resources, J.D.C.; Software, M.S.; Supervision, M.R.; Validation, S.A.; Visualization, T.M.; Writing—original draft, M.R.; Writing—review \& editing, D.L.

Funding: This research was funded by National Natural Science Foundation China (No. 11571140 and 11671077), Faculty of Science, Jiangsu University, Zhenjiang, China.

Acknowledgments: This research was funded by National Natural Science Foundation China (No. 11571140 and 11671077), Faculty of Science, Jiangsu University, Zhenjiang, China.

Conflicts of Interest: Authors have no conflict of interest regarding this publication.

\section{Nomenclature}

U

$V$

$U_{w}$

$U_{e}$

$M$

$\rho_{f}, \rho_{s}$

$\phi$

$\tau_{w}$

$T_{\infty}$

$\lambda$

$\sigma_{n f}$

$\gamma$

$k_{f}$

$k_{s}$

$\delta$

$q_{r}$

$h_{f}$

$C_{f}$

$N u_{x}$

$T$

$T_{f}$

$h_{S}$

$\mu_{n f}$

$R e_{x}$

$k^{*}$

$\sigma^{*}$ along $x$-axis fluid velocity $[\mathrm{m} / \mathrm{s}]$

along $r$-axis fluid velocity $[\mathrm{m} / \mathrm{s}]$

Stretching velocity $[\mathrm{m} / \mathrm{s}]$

Free stream velocity $[\mathrm{m} / \mathrm{s}]$

Magnetic parameter

Density of fluid and solid particle respectively $\left[\mathrm{kg} / \mathrm{m}^{3}\right]$

nanofluid volume fraction

surface shear stress $\left[\mathrm{N} / \mathrm{m}^{2}\right]$

Ambient temperature [K]

conjugate parameter

electric conductivity of fluid and nanofluid respectively $[\mathrm{S} / \mathrm{m}]$

curvature parameter

thermal conductivities of fluid $\left[\mathrm{J} / \mathrm{mKs}^{n-2}\right]$

thermal conductivities of nanomaterial $\left[\mathrm{J} / \mathrm{mKs}^{n-2}\right]$

ratio of mass diffusion coefficients

radiative heat flux $\left[\mathrm{kg} / \mathrm{m}^{2}\right]$

convective heat transfer coefficient

Skin friction coefficient

Nusselt number

Temperature [K]

convective fluid temperature [K]

heat transfer coefficient

Nanofluid dynamic viscosity $\left[\mathrm{kg} / \mathrm{ms}^{n-2}\right]$

local Reynolds number

mean absorption coefficient

Stefan-Boltzmann constant 


$\begin{array}{ll}D_{A}, D_{B} & \text { diffusion coefficients }\left[\mathrm{m}^{2} / \mathrm{s}\right] \\ q_{w} & \text { surface heat flux }\left[\mathrm{W} / \mathrm{m}^{2}\right] \\ S_{c} & \text { Schmidt number } \\ k_{c}, k_{s} & \text { Rate constants } \\ K^{*} & \text { Radiation parameter } \\ \mu_{f} & \text { Fluid dynamic viscosity }\left[\mathrm{kg} / \mathrm{ms}^{n-2}\right] \\ \alpha_{n f} & \text { Nanofluid thermal diffusivity }\left[\mathrm{m}^{2} / \mathrm{s}^{n-2}\right] \\ k_{1} & \text { Strength of homogeneous reaction } \\ v_{n f} & \text { nanofluid kinematic viscosity }\left[\mathrm{m}^{2} / \mathrm{s}\right] \\ k_{2} & \text { strength of heterogeneous reaction } \\ R & \text { radius of cylinder }[\mathrm{m}] \\ B & \text { Magnetic field strength }[\text { A } / \mathrm{m}] \\ N_{r} & \text { temperature ratio parameter } \\ A_{1}, B_{1} & \text { concentrations of chemical species } a, b \\ n & \text { Nonlinearity exponent }\end{array}$

\section{References}

1. Choi, S.U.S. Enhancing conductivity of fluids with nanoparticles, ASME Fluid Eng. Division 1995, 231, 99-105.

2. Minkowycz, W.J.; Sparrow, E.M.; Abraham, J.P. (Eds.) Nanoparticle Heat Transfer and Fluid Flow; CRC Press: Boca Raton, FL, USA, 2012; Volume 4.

3. Kakac, S.; Pramuanjaroenkij, A. Review of convective heat transfer enhancement with nanofluids. Int. J. Heat Mass Transf. 2009, 52, 3187-3196. [CrossRef]

4. Li, Z.; Sheikholeslami, M.; Shafee, A.; Ramzan, M.; Kandasamy, R.; Al-Mdallal, Q.M. Influence of adding nanoparticles on solidification in a heat storage system considering radiation effect. J. Mol. Liq. 2019, 273, 589-605. [CrossRef]

5. Sheikholeslami, M.; Shafee, A.; Ramzan, M.; Li, Z. Investigation of Lorentz forces and radiation impacts on nanofluid treatment in a porous semi annulus via Darcy law. J. Mol. Liq. 2018, 272, 8-14. [CrossRef]

6. Lu, D.; Ramzan, M.; Ahmad, S.; Chung, J.D.; Farooq, U. Upshot of binary chemical reaction and activation energy on carbon nanotubes with Cattaneo-Christov heat flux and buoyancy effects. Phys. Fluids 2017, 29, 123103. [CrossRef]

7. Ramzan, M.; Ullah, N.; Chung, J.D.; Lu, D.; Farooq, U. Buoyancy effects on the radiative magneto Micropolar nanofluid flow with double stratification, activation energy and binary chemical reaction. Sci. Rep. 2017, 7, 12901. [CrossRef] [PubMed]

8. Suleman, M.; Ramzan, M.; Zulfiqar, M.; Bilal, M.; Shafee, A. Entropy analysis of 3D non-Newtonian MHD nanofluid flow with nonlinear thermal radiation past over exponential stretched surface. Entropy 2018, 20, 930. [CrossRef]

9. Farooq, U.; Lu, D.C.; Ahmed, S.; Ramzan, M.; Chung, J.D.; Chandio, F.A. Computational analysis for mixed convective flows of viscous fluids with nanoparticles. J. Therm. Sci. Eng. Appl. 2019, 11, 021013. [CrossRef]

10. Ramzan, M.; Sheikholeslami, M.; Saeed, M.; Chung, J.D. On the convective heat and zero nanoparticle mass flux conditions in the flow of 3D MHD Couple Stress nanofluid over an exponentially stretched surface. Sci. Rep. 2019, 9, 562. [CrossRef] [PubMed]

11. Ramzan, M.; Bilal, M.; Chung, J.D.; Mann, A.B. On MHD radiative Jeffery nanofluid flow with convective heat and mass boundary conditions. Neural Comput. Appl. 2019, 30, 2739-2748. [CrossRef]

12. Ramzan, M.; Sheikholeslami, M.; Chung, J.D.; Shafee, A. Melting heat transfer and entropy optimization owing to carbon nanotubes suspended Casson nanoliquid flow past a swirling cylinder-A numerical treatment. AIP Adv. 2018, 8, 115130. [CrossRef]

13. Lu, D.; Ramzan, M.; Ullah, N.; Chung, J.D.; Farooq, U. A numerical treatment of radiative nanofluid 3D flow containing gyrotactic microorganism with anisotropic slip, binary chemical reaction and activation energy. Sci. Rep. 2017, 7, 17008. [CrossRef] [PubMed] 
14. Ramzan, M.; Bilal, M.; Kanwal, S.; Chung, J.D. Effects of variable thermal conductivity and non-linear thermal radiation past an Eyring Powell nanofluid flow with chemical reaction. Commun. Theor. Phys. 2017, 67, 723. [CrossRef]

15. Muhammad, T.; Lu, D.C.; Mahanthesh, B.; Eid, M.R.; Ramzan, M.; Dar, A. Significance of Darcy-Forchheimer porous medium in nanofluid through carbon nanotubes. Commun. Theor. Phys. 2018, 70, 361. [CrossRef]

16. Azam, M.; Shakoor, A.; Rasool, H.F.; Khan, M. Numerical simulation for solar energy aspects on unsteady convective flow of MHD Cross nanofluid: A revised approach. Int. J. Heat Mass Transf. 2019, 131, 495-505. [CrossRef]

17. Sheikholeslami, M.; Kataria, H.R.; Mittal, A.S. Effect of thermal diffusion and heat-generation on MHD nanofluid flow past an oscillating vertical plate through porous medium. J. Mol. Liq. 2018, 257, 12-25. [CrossRef]

18. Makinde, O.D.; Animasaun, I.L. Bioconvection in MHD nanofluid flow with nonlinear thermal radiation and quartic autocatalysis chemical reaction past an upper surface of a paraboloid of revolution. Int. J. Therm. Sci. 2016, 109, 159-171. [CrossRef]

19. Lu, D.; Ramzan, M.; Bilal, M.; Chung, J.D.; Farooq, U. A numerical investigation of 3D MHD rotating flow with binary chemical reaction, activation energy and non-Fourier heat flux. Commun. Theor. Phys. 2018, 70, 089. [CrossRef]

20. Rana, P.; Shukla, N.; Gupta, Y.; Pop, I. Analytical prediction of multiple solutions for MHD Jeffery-Hamel flow and heat transfer utilizing KKL nanofluid model. Phys. Lett. A 2019, 383, 176-185. [CrossRef]

21. Ramzan, M.; Bilal, M.; Chung, J.D.; Lu, D.; Farooq, U. Impact of generalized Fourier's and Fick's laws on MHD 3D second grade nanofluid flow with variable thermal conductivity and convective heat and mass conditions. Phys. Fluids 2017, 29, 093102. [CrossRef]

22. Yuan, M.; Mohebbi, R.; Rashidi, M.M.; Yang, Z.; Sheremet, M.A. Numerical study of MHD nanofluid natural convection in a baffled U-shaped enclosure. Int. J. Heat Mass Transf. 2019, 130, 123-134.

23. Rahimah, J.; Nazar, R.; Pop, I. Magnetohydrodynamic boundary layer flow and heat transfer of nanofluids past a bidirectional exponential permeable stretching/shrinking sheet with viscous dissipation effect. J. Heat Transf. 2019, 141, 012406.

24. Benos, L.; Sarris, I.E. Analytical study of the magnetohydrodynamic natural convection of a nanofluid filled horizontal shallow cavity with internal heat generation. Int. J. Heat Mass Transf. 2019, 130, 862-873. [CrossRef]

25. Sajjadi, H.; Delouei, A.A.; Izadi, M.; Mohebbi, R. Investigation of MHD natural convection in a porous media by double MRT lattice Boltzmann method utilizing MWCNT-Fe $\mathrm{O}_{4} /$ water hybrid nanofluid. Int. J. Heat Mass Transf. 2019, 132, 1087-1104. [CrossRef]

26. Zhao, G.; Wang, Z.; Jian, Y. Heat transfer of the MHD nanofluid in porous microtubes under the electrokinetic effects. Int. J. Heat Mass Transf. 2019, 130, 821-830. [CrossRef]

27. Ibrahim, M.G.; Hasona, W.M.; ElShekhipy, A.A. Concentration-dependent viscosity and thermal radiation effects on MHD peristaltic motion of Synovial Nanofluid: Applications to rheumatoid arthritis treatment. Comput. Methods Programs Biomed. 2019, 170, 39-52. [CrossRef] [PubMed]

28. Merkin, J.H. Natural-convection boundary-layer flow on a vertical surface with Newtonian heating. Int. J. Heat Fluid Flow 1994, 15, 392-398. [CrossRef]

29. Kamran, M.; Wiwatanapataphee, B. Chemical reaction and Newtonian heating effects on steady convection flow of a micropolar fluid with second order slip at the boundary. Eur. J. Mech. B/Fluids 2018, 71, 138-150. [CrossRef]

30. Mehmood, R.; Rana, S.; Nadeem, S. Transverse thermopherotic MHD Oldroyd-B fluid with Newtonian heating. Results Phys. 2018, 8, 686-693. [CrossRef]

31. Shafiq, A.; Hammouch, Z.; Sindhu, T.N. Bioconvective MHD flow of tangent hyperbolic nanofluid with Newtonian heating. Int. J. Mech. Sci. 2017, 133, 759-766. [CrossRef]

32. Khan, A.; Khan, D.; Khan, I.; Ali, F.; Karim, F.U.; Imran, M. MHD flow of Sodium Alginate-based Casson type nanofluid passing through a porous medium with Newtonian heating. Sci. Rep. 2018, 8, 8645. [CrossRef] [PubMed]

33. El-Hakiem, M.A.; Ramzan, M.; Chung, J.D. A numerical study of magnetohydrodynamic stagnation point flow of nanofluid with Newtonian heating. J. Comput. Theor. Nanosci. 2016, 13, 8419-8426. [CrossRef] 
34. Ramzan, M.; Yousaf, F. Boundary layer flow of three-dimensional viscoelastic nanofluid past a bi-directional stretching sheet with Newtonian heating. AIP Adv. 2015, 5, 057132. [CrossRef]

35. Shehzad, S.A.; Hussain, T.; Hayat, T.; Ramzan, M.; Alsaedi, A. Boundary layer flow of third grade nanofluid with Newtonian heating and viscous dissipation. J. Cent. South Univ. 2015, 22, 360-367. [CrossRef]

36. Ramzan, M. Influence of Newtonian heating on three dimensional MHD flow of couple stress nanofluid with viscous dissipation and Joule heating. PLoS ONE 2015, 10, e0124699. [CrossRef] [PubMed]

37. Upreti, H.; Pandey, A.K.; Kumar, M. MHD flow of Ag-water nanofluid over a flat porous plate with viscous-Ohmic dissipation, suction/injection and heat generation/absorption. Alex. Eng. J. 2018, 57, 1839-1847. [CrossRef]

38. Qasim, M.; Khan, Z.H.; Khan, W.A.; Shah, I.A. MHD boundary layer slip flow and heat transfer of ferrofluid along a stretching cylinder with prescribed heat flux. PLoS ONE 2014, 9, e83930. [CrossRef] [PubMed]

(C) 2019 by the authors. Licensee MDPI, Basel, Switzerland. This article is an open access article distributed under the terms and conditions of the Creative Commons Attribution (CC BY) license (http:/ / creativecommons.org/licenses/by/4.0/). 